\title{
BNT162b2 Vaccine: Possible Codons Misreading, Errors in Protein Synthesis and Alternative Splicing's Anomalies
}

\section{Kira Smith}

Department of Clinical and Experimental Medicine, University of Eastern Piedmont, Norova, Italy

\begin{abstract}
BNT162b2 vaccine against Covid-19 is composed of an RNA having 4284 nucleotides, divided into 6 sections, which bring the information to create a factory of S Spike proteins, the ones used by Sars-CoV-2 (Covid-19) to infect the host. After that, these proteins are directed outside the cell, triggering the immune reaction and antibody production.
\end{abstract}

The problem is the heavy alteration of the mRNA: Uracil is replaced to fool the immune system with $\Psi$ (Pseudouridine); the letters of all codon triplets are replaced by a $\mathrm{C}$ or a $\mathrm{G}$, to extremely increase the speed of protein synthesis; replacement of some amino acids with Proline; addition of a sequence (3'-UTR) with unknown alteration.

These impairments could cause strong doubts about the presence of codon usage errors. An eventual mistranslation has consequences on the pathophysiology of a variety of diseases. In addition, mRNA injected is a pre-mRNA, which can lead to the multiple mature mRNAs; these are alternative splicing anomalies, direct source of serious long-term harm on the human health.

In essence, what will be created may not be identical with protein S Spike: just an error in translational decoding, codons misreading, production of different amino acids, then proteins, to cause serious long-term damage to human health, despite the DNA is not modified, being instead in the cell nucleus and not in the cytoplasm, where the modified mRNA arrives.

However, in this case, the correlation between speed of synthesis and protein expression with synthesis errors, as well as the mechanism that could affect the translation of the sequence remain obscure, many trials have not yet been performed.

Keywords: Covid-19; Coronavirus; BNT162b2; Alternative splicing; Misreading; Codons

\section{INTRODUCTION}

\section{How vaccine works}

BioNTec/Pfizer's SARs CoV-2 (Covid-19) vaccine called BNT162b2, but also called Tozinameran, or Comirnaty, contains about $30 \mathrm{mcg}$ of RNA, which is injected into a lipid sphere inside the human body, specifically inside the cytoplasm of cells, but outside the nucleus (where the DNA is located); this RNA has modified genetic information (hence modRNA), i.e. an mRNA (messenger RNA) containing instructions to set in place a factory of proteins, clones of the protein S Spike, i.e. the protein (and only the protein, not the whole virus) used by Covid-19 to enter the host and infect it. Once they are serially produced by the ribosomes, they are transported outside the cell, beyond the lipid coating; in this way the immune system identifies these proteins as cell invaders and attacks them, through the production of antibodies. This is why it is not conceivable that the vaccine induces Covid-19, or that it modifies human DNA.

\section{Protein synthesis}

Translation is generally divided into three phases: Beginning, lengthening, and ending. The ribosome binds to the mRNA at the start codon; the polypeptide chain elongates in one direction of ribosome movement, by successive addition of amino acids; when a

Correspondence to: Kira Smith, Department of Clinical and Experimental Medicine, University of Eastern Piedmont, Norova, Italy, E-mail: kirasmith@mil-med.com

Received: January 15, 2021; Accepted: January 29, 2021; Published: February 05, 2021

Citation: Smith K (2021) BNT162b2 Vaccine: Possible Codons Misreading, Errors in Protein Synthesis and Alternative Splicing's Anomalies. J Antivir Antiretrovir. 13:210.

Copyright: () 2021 Smith K. This is an open-access article distributed under the terms of the Creative Commons Attribution License, which permits unrestricted use, distribution, and reproduction in any medium, provided the original author and source are credited. 
Stop codon is found, the polypeptide is released and the ribosome dissociates.

\section{Errors in sequence assembly and translation}

Converting the sequence of mRNA into a polypeptide depends on transfer RNA (tRNA) to carry amino acids to the ribosome. At ribosomes, tRNA pairs with mRNA by complementary base pairing between mRNA codon nucleotides and tRNA anticodon nucleotides. Once the correct tRNA is bound by a codon, it transfers its amino acid to the end of a growing polypeptide chain.

Deciphering mRNA codons by transfer RNAs (tRNAs) in the ribosome involves Watson-Crick base pairing.

The general error rates of genomic replication (about $10^{-8}$ ) are estimated to be approximately 10,000-fold lower than those of protein synthesis (about $10^{-4}$ ), and thus in most instances mRNA translation is the key process contributing to inaccuracy of the cellular proteome. The discrepancy between error rates in DNA replication and mRNA translation may partially relate to the fact that DNA replication occurs at the level of individual nucleotides (involving $4^{1}=4$ possible permutations), whereas the translation machinery interprets mRNA codons in triplets (involving $4^{3}=64$ possible permutations) [1].

The efficiency of mRNA decoding machinery is also essentially regulated by codon usage bias that is distinguished by over or underrepresented synonymous codons. Accordingly, optimizing of tRNA wobble and codon usage in mRNA can substantially enhance translation efficiency and accuracy [1].

Pre or post-mRNA translation may indirectly introduce errors of protein synthesis during transcription and posttranslational processing. However, the translation machinery can directly contribute to mistranslation by tRNA misdecoding (leading to misincorporation or stop-codon read-through), tRNA misacylation (leading to wrong tRNA amino acid coupling), codon reassignment or ribosomal translocation-provoked frame shifts [1].

\section{INVESTIGATION METHOD}

\section{Genetic sequence analysis}

The vaccine is composed of 4284 nucleotides, divided into 6 sections: Cap is the beginning of the sequence, which opens with the two GA nucleotides, falsely indicating that the mRNA comes from the human cell and thus be accepted; 5 ' indicates the direction to be followed for translation, while UTR indicates the area where the ribosome must rest in order to manufacture proteins. In this section, the $U$ of Uracil has been replaced with one molecule of 1-methyl-3'-pseudouridine, indicated with the character $\Psi$, to bypass the immune system and prevent the degradation of the mRNA that has just entered; however, this is a factor that can lead to errors in protein production. Multiple $\Psi$ synthases are involved in the modification of specific positions, and defects in several of them are linked to human disease [2].

Then there is the sig section, called the extended startup sequence of the S-glycoprotein signaling peptide, whose information is needed to guide the newly formed protein out of the cell via the endoplasmic reticulum; here too are put in place changes to the triplets of nucleotides to make the RNA accepted by the immune system, changing some letters, that make up the information, with others (usually in $3^{\text {rd }}$ position, "wobble"), apparently "harmless synonyms" (mainly by increasing the number of letters $\mathrm{C}$ and $\mathrm{G}$, which encode the speed of protein synthesis). However, while they specify identical amino acids, the two synonyms are not precisely the same, at least when it comes to the act of translation. Mechanistic studies show that there are subtle but significant differences in how each interacts with its corresponding transfer RNA (tRNA), differences that affect both the speed and the accuracy of translation [3]. While it is true that 3 letters form a codon and more than one codon encodes for the same amino acid, it is also true that by disproportionately increasing the rate of protein production, could be risks of serious translational errors.

Also the characters that compose the sequence related to the construction of the real Spike protein S protein_mut have been altered with more $\mathrm{C}$ and $\mathrm{G}$ that was possible to add, respecting the synonyms in the standard genetic code table, with substitution of the Amino Acids Lysine (AAA) and Valine (GUU) with Proline (CUU), to prevent the constructed protein to collapse on itself. At the end of this sequence there are 2 stop codons. It's not fully proven that the same elements will be formed with this substitution and won't be misreading errors.

3'-UTR (Untranslated Region 3 First): It should indicate the direction of translation of the sequence and improve protein synthesis, however many of its functions remain unknown; therefore it is impossible to ascertain its safety. What is known as stated by WHO and is the following sentence: The 3' UTR for the BioNTech/Pfizer vaccine was taken from "the Amino-terminal Enhancer of Split (AES) mRNA and the mitochondrial encoded $12 \mathrm{~S}$ ribosomal RNA".

Poly (A): We then reach the end of the sequence and encounter $30 \mathrm{~A}$ 's, then a 10-nucleotide GCAUAUGACU linkage, followed by another $70 \mathrm{~A}$ 's, since each mRNA can be reused by the organism multiple times.

When the A's run out, the mRNA is degraded.

All of these are proprietary modifications to increase protein expression, of which nothing is known about the actual translation implemented by the organism.

\section{Alternative splicing and other errors}

Another related problem is that the same pre-mRNA can give rise to different mature mRNAs, and therefore to slightly different proteins (anomalies of alternative splicing). An alteration in the process of protein synthesis has been found to be the cause of the development and growth of some cancers, and other diseases, without altering the DNA in any way.

All splicing events identified in the three PHT series genes involve the loss of the messenger sequence reading frame, and the introduction of a Premature Termination Codon (PTC) always located more than 50-55 nucleotides upstream of the last exonexon junction, which makes the alternative transcripts targets of the NMD (Nonsense-Mediated mRNA Decay) surveillance system. For human and rat slc15a4/PHT1, this was demonstrated by NMD inhibition experiments in different cell lines, in which the expression of alternative variants to canonical transcripts was always stabilized following inhibition [4].

\section{Possible long-term risks on human health}

We can say that besides not being optimized, the sequence presents 
strong doubts about the presence of codon usage errors. It's possible to hypothesize that an excessive alteration aimed at an extreme increase in protein expression may be the source of errors in the assembly of the mRNA gene sequence.

Altering tRNA availability can lead to neurodegenerative diseases and up regulation of specific tRNAs drives metastasis by enhancing stability of transcripts enriched in their cognate codons [5].

Mistranslation has very serious consequences on the pathophysiology of a variety of diseases, including multiple sclerosis, neurodegeneration, mitochondrial myopathy, encephalopathy, lactic acidosis, stroke-like episodes, Parkinson's disease, and cancer (genesis, growth acceleration and metastasis) [6].

The correlation between the speed of protein synthesis, increased by $100 \%$, with the translation errors of the sequence, as well as the mechanism that affects the production of amino acids remain in this case for now obscure, being many trials have not yet been performed.

\section{CONCLUSION}

Basically it can be said that the code of the total sequence is intrinsically altered in an unbalanced way, too much compared to the natural viral counterpart, and too much to be able to say that the human organism reproduces exactly the S Spike proteins, equal to each other, thus risking to bring serious damage to human health in the long term, in addition to failure to be efficient in immunization.

What will be produced from that sequence is far from well defined, but it is written in the genes of each individual, by ribosomal profile, how it will be translated and what will be produced, thus the benefits or damages that will be caused.

\section{REFERENCES}

1. Ou X, Cao J, Cheng A, Peppelenbosch MP, Pan Q. Errors in translational decoding: tRNA wobbling or misincorporation? PLOS Genet. 2019; 15(3): e1008017.

2. Khonsari B, Klassen R. Impact of pus1 pseudouridine synthase on specific decoding events in Saccharomyces cerevisia. Biomolecules. 2020; 10(5):729.

3. Robinson R. Which codon synonym is best? It may depend on what's on the menu. PLOS Biol. 2014; 12(12): e1002014.

4. Andries O. mRNA Modification and delivery strategies towards the establishment of a platform for safe and effective gene therapy. Universitiet Ghent. 2015.

5. Qiushuang Wu, Medina SG, Kushawah G, DeVore ML, Castellano LA, Hand JM, et al. Translation affects mRNA stability in a codondependent manner in human cells. eLife. 2019;.8:e45396.

6. Santos M, Pereira PM, Varanda AS, Carvalho J, Azevedo M, Mateus $\mathrm{DD}$, et al. Codon misreading tRNAs promote tumor growth in mice. RNA Biol. 2018; 15(6):773-786. 